\title{
Kurz berichtet
}

Online publiziert: 9. September 2019

(c) Springer-Verlag GmbH Deutschland, ein Teil von Springer Nature 2019

\section{Hirnströme direkt am Arbeitsplatz messen: Workshop für kognitive Neuroergonomie}

Vom 18.-19.06. hat ein Qualifizierungsworkshop am IfADo zum Thema kognitive Neuroergonomie stattgefunden. Der Workshop richtete sich an wissenschaftliche Mitarbeiterinnen und Mitarbeiter, die Mitglied in der Gesellschaft für Arbeitswissenschaft sind.

Prof. Edmund Wascher, Leiter des Forschungsbereichs Ergonomie, gab eine Einführung in das Thema kognitive Neuroergonomie. Für die experimentelle Neuropsychologie besteht eine elementare Technik in der Elektroenzephalografie (EEG), die bereits vor hundert Jahren entwickelt wurde. Zahlreiche EEG-Studien geben Aufschluss darüber, wie das Gehirn des Menschen Informationen verarbeitet. Die Methode hatte allerdings bisher zwei Haken: Sie ist empfindlich gegenüber Bewegungen und sie ist kabelgebunden und dementsprechend auch ortsgebunden.

Neue Methoden setzen hier an und holen das EEG raus aus dem Labor hinein in die reale Welt, um relevante $\mathrm{Si}$ tuationen messen zu können. Gerade für angewandte Fragestellungen im arbeitswissenschaftlichen Kontext machen EEG-Messungen direkt am Arbeitsplatz Sinn. Seit wenigen

Quelle: https://www.ifado.de/blog/2019/06/19/kognitiveneuroergonomie/
Jahren sind mobile EEG-Geräte erhältlich, deren Messgenauigkeit aktuell auf Herz und Nieren geprüft wird.

Im Rahmen des Workshops erläuterte Julian Reiser, wissenschaftlicher IfADo-Mitarbeiter der Ergonomie, die Chancen und Herausforderungen verschiedener mobiler EEG-Methoden. Die grundsätzliche Frage, wie die Signale ausgewertet und interpretiert werden können, konkretisierte Dr. Stefan Arnau, ebenso wissenschaftlicher IfADo-Mitarbeiter der Ergonomie. Dabei besprach er insbesondere die neurologischen Korrelate von Belastung und mentaler Ermüdung.

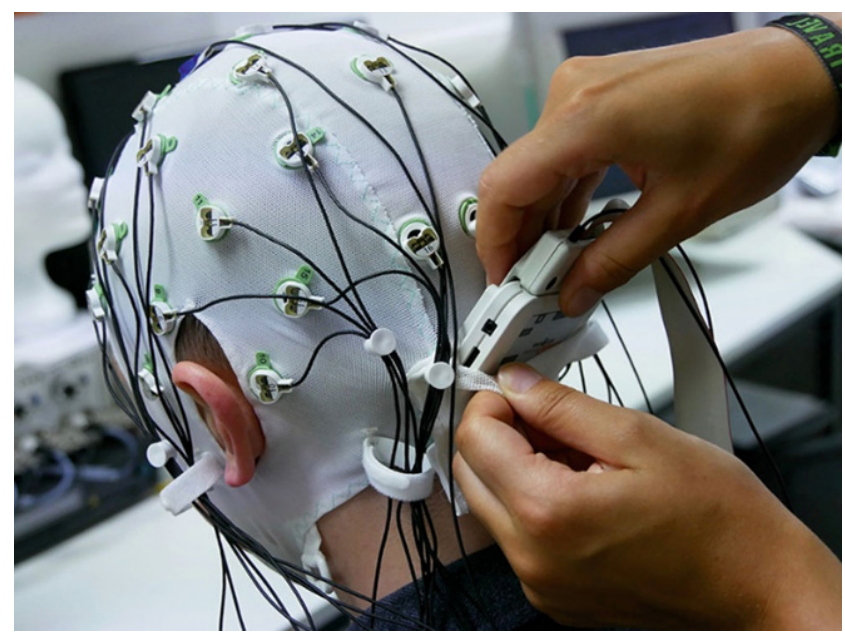

Verkabelung eines Probanden für EEG-Messung 